\title{
THE ISOMETRY GROUPS OF MANIFOLDS AND THE AUTOMORPHISM GROUPS OF DOMAINS
}

\author{
RITA SAERENS AND WILLIAM R. ZAME
}

\begin{abstract}
We prove that every compact Lie group can be realized as the (full) automorphism group of a strictly pseudoconvex domain and as the (full) isometry group of a compact, connected, smooth Riemannian manifold.
\end{abstract}

1. Introduction. Given an instance of a mathematical structure, we are led to ask for its group of symmetries; i.e., the group of structure-preserving selftransformations. The inverse problem is to ask which groups can occur as the group of symmetries of a given kind of structure.

In this paper, we consider two such inverse problems:

(a) Which groups occur as the (full) group of isometries of a compact, connected Riemannian manifold?

(b) Which groups occur as the (full) group of (biholomorphic) automorphisms of a strictly pseudoconvex domain?

Definitions will be given below.

We recall that Myers and Steenrod [22] have shown that the isometry group of a Riemannian manifold is a Lie group. This group is compact if the manifold is compact. Cartan [7] has shown that the automorphism group of a bounded domain (i.e., a connected, open subset of $\mathbf{C}^{n}$ ) is a (real) Lie group. If the domain is strictly pseudoconvex and not biholomorphically equivalent to the ball, Wong [26] and Rosay [24] have shown that its automorphism group is compact. (The automorphism group of the ball in $\mathbf{C}^{n}$ is the special unitary group associated with a certain indefinite inner product on $\mathrm{C}^{n+1}$, see [18].)

Thus our questions are completely answered by the following results.

THEOREM 1. Every compact Lie group can be realized as the (full) group of isometries of a compact Riemannian manifold.

THEOREM 2. Ever compact Lie group can be realized as the (full) group of automorphisms of a strictly pseudoconvex domain.

(We stress that we are interested in the full group of isometries or automorphisms. It is quite easy to realize a compact Lie group as a subgroup of such groups.)

These two results, which on the face of it seem unrelated, are in fact connected. This may not seem surprising; after all, the use of techniques from Riemannian

Received by the editors May 26, 1986.

1980 Mathematics Subject Classification (1985 Revision). Primary 32F15, 32M05, 53C20.

We are thankful to Frank Beatrous, Dan Burns, Mike Cowen, Jim Faran, and Robert Greene for helpful conversations, and to the Department of Mathematics of the University of Washington for hospitality in the summer of 1983 , when this research was done.

Financial support from the National Science Foundation is gratefully acknowledged. 
geometry to study problems in complex analysis is by now well established. In particular, Bedford [1] and Greene and Krantz [13, 14] have studied automorphism groups of domains by passing to appropriate Riemannian manifolds. This suggests trying to solve the Riemannian problem first. A natural attempt runs as follows. First construct a compact Riemannian manifold $M$, with metric $\gamma$, on which the compact Lie group $G$ acts by isometries; then perturb $\gamma$ in such a way that the isometries in $G$ are preserved and all the other ones are eliminated. To do this, one is led to construct a $G$-invariant metric $\gamma^{\prime}$ on $M$ with the following property:

If $x, y \in M$ and $y$ does not belong to the $G$-orbit of $x$, then the curvature of $\gamma^{\prime}$ is different at $x$ and at $y$.

This can always be accomplished (by combining methods of Ebin [10] with a transversality argument) provided the dimension of $M$ is sufficiently large (compared with the dimension of $G$ ). Unfortunately, this property only guarantees that every isometry of $\gamma^{\prime}$ respects the orbit structure of $G$; it does not guarantee that the isometries of $\gamma^{\prime}$ actually belong to $G$.

However, similar ideas do work in the complex-analytic case. We use the local holomorphic curvature invariants of the boundary of a domain, à la Burns, Shnider, and Wells [6] (instead of the curvature of a Riemannian metric) to construct a strictly pseudoconvex domain $D$ with the property that all the automorphisms of $D$ leave $G$-orbits invariant. In the complex analytic case, this rigidity turns out to be enough to guarantee that all the automorphisms of $D$ actually belong to $G$; i.e., $G$ is realized as the full automorphism group of $D$.

The solution to the Riemannian problem is obtained from the solution to the complex-analytic one. To do so, we construct-following an idea of Greene and Krantz [13]-a special metric on the double $M$ of $D$. The construction guarantees that the isometry group of $M$ is a finite extension of $G$ and that every isometry of $M$ which is not in $G$ moves an orbit of $G$. We can then slightly perturb the metric on $M$ to eliminate the unwanted isometries, and realize the group $G$ as the full isometry group.

The remainder of the paper is organized in the following way. In $\S 2$, we collect the information we need about strictly pseudoconvex domains, boundary behavior of biholomorphic mappings and Chern-Moser invariants. The proof of Theorem 2 is given in $\S 3$. In $\S 4$, we collect some information about Riemannian geometry and the Bergman metric. The proof of Theorem 1 is given in $\S 5$.

After this paper was written, we received preprints of two papers by Bedford and Dadok $[\mathbf{2}, \mathbf{3}]$ who have obtained the same results by different methods.

2. Complex-analytic background. In this section we collect the information we shall need from the theory of several complex variables. In the interest of brevity, we shall give it in the form most suited to our context. For further information, see the book of Krantz [18], the survey paper by Burns and Shnider [5], and the papers cited below.

Let $U$ be a domain (bounded, connected open set) in $\mathbf{C}^{n}(n \geq 2)$. A smooth (i.e., $C^{\infty}$ ) function $\varphi: U \rightarrow \mathbf{R}$ is strictly plurisubharmonic if the Levi 2 -form

$$
L \varphi=\sum \frac{\partial^{2} \varphi}{\partial z_{i} \partial \bar{z}_{j}} d z_{i} \wedge d \bar{z}_{j}
$$


is positive definite. A domain $D$ with smooth (i.e., $C^{\left.\infty_{-}\right)}$boundary is strictly pseudoconvex if there is a domain $U$ containing the boundary $b D$ of $D$ and a strictly plurisubharmonic function $\varphi$ on $U$ such that $D \cap U=\{\varphi<0\}$, and $d \varphi$ is nonvanishing on $b D$.

For our purpose, the crucial fact about strictly pseudoconvex domains is the following result of Fefferman $[\mathbf{1 1}]$.

THEOREM. Let $D_{1}$ and $D_{2}$ be strictly pseudoconvex domains with smooth boundaries, and let $h: D_{1} \rightarrow D_{2}$ be a biholomorphic mapping (i.e., a holomorphic mapping with a holomorphic inverse). Then $h$ extends to a $C^{\infty}$-diffeomorphism

$$
\hat{h}: \bar{D}_{1} \rightarrow \bar{D}_{2} \text {. }
$$

Fefferman's theorem reduces many questions about biholomorphic mappings between strictly pseudoconvex domains $D_{1}$ and $D_{2}$ to corresponding questions about CR-diffeomorphisms between their boundaries $b D_{1}$ and $b D_{2}$. (A CR-mapping $f: b D_{1} \rightarrow b D_{2}$ is a smooth mapping such that the restriction of $(d f)_{x}$ to the maximal complex tangent space of $b D_{1}$ at $x$ is complex-linear for each $x \in b D_{1}$ (see [6]). This is equivalent to requiring $f$ to satisfy the tangential Cauchy-Riemann equations.) The study of the existence of CR-diffeomorphisms goes back to Poincaré [23]. Obstructions to the existence of such mappings have been found by Cartan [7] and Chern and Moser [8]. These obstructions are invariants of the local holomorphic curvature of the boundary hypersurfaces. Using Moser's normal form, Burns, Shnider and Wells [6] constructed scalar curvature invariants in a form which is convenient for our use.

To describe these invariants, it is convenient to work in $\mathbf{C}^{n+1}$, with $n \geq 2$. We write $\mathbf{C}^{n+1}=\mathbf{C}^{n} \times \mathbf{C}$, where $z_{1}, \ldots, z_{n}$ are the variables in $\mathbf{C}^{n}$ and $w=u+i v$ is the last variable. We consider the hypersurface $\{\varphi=0\}$ near the origin (assuming that $\varphi(0)=0)$. After a formal power series transformation, the defining function $\varphi$ may be written in normal form, i.e.,

$$
\varphi=u-\langle z, z\rangle-\sum_{p, q \geq 2} N_{p, q}
$$

where $\langle$,$\rangle is the standard inner product on \mathbf{C}^{n}$, and $N_{p, q}$ is a polynomial of type $(p, q)$ in $z$ whose coefficients are formal power series in $u$; i.e.,

$$
N_{p, q}=\sum N_{\alpha_{1} \ldots \alpha_{p}, \beta_{1} \ldots \beta_{q}}(u) z^{\alpha_{1}} \cdots z^{\alpha_{p}} \bar{z}^{\beta_{1}} \cdots \bar{z}^{\beta_{q}},
$$

with

$$
N_{\alpha_{1} \ldots \alpha_{p}, \beta_{1} \ldots \beta_{q}}(u)=\sum_{j \geq 0} N_{\alpha_{1} \ldots \alpha_{p}, \beta_{1} \ldots \beta_{q}}^{j} u^{j}
$$

(There are certain normalizations, which we omit.) The origin is called an umbilic point if $\sum\left|N_{\alpha_{1} \alpha_{2}, \beta_{1} \beta_{2}}^{0}\right|^{2}=0$; otherwise, the origin is nonumbilic. (These notions are independent of the normal form of $\varphi$ and are invariant under biholomorphic transformations.) If the origin is nonumbilic, certain other normalizations may be made; when this is done, the representation of $\varphi$ is said to be in restricted normal form. With $\varphi$ in restricted normal form the curvature invariants we seek are defined for $j \geq 0, p \geq q \geq 2, p \geq 3$ by

$$
K_{p, q}^{j}=\sum\left|N_{\alpha_{1} \ldots \alpha_{p}, \beta_{1} \ldots \beta_{q}}^{j}\right|^{2} .
$$


(The normalizations are irrelevant for our purposes; for details, see [6].)

Burns, Shnider, and Wells show that the restricted normal form is essentially unique. For our purposes, this is irrelevant; all we shall need is that the scalars $K_{p, q}^{j}$ are invariants of the local CR-structure of the hypersurface $\{\varphi=0\}$ near the origin. More precisely, if $\{\psi=0\}$ is another such hypersurface (with $\varphi, \psi$ strictly plurisubharmonic and $\varphi(0)=0, \psi(0)=0$ ), and the origin is nonumbilic in each hypersurface, then the existence of a CR-diffeomorphism $f$ of a neighborhood of the origin in $(\varphi=0)$ with a neighborhood of the origin in $\{\psi=0\}$ (sending the origin to itself) implies that $K_{p, q}^{j}(\varphi)=K_{p, q}^{j}(\psi)$.

Three remarks need to be made. The first is that these curvature invariants are only defined at nonumbilic points. However, when $n \geq 3$ the set of umbilic points is generically empty (see [6, p. 246]). In the dimensions in which we work, this means that we shall be able to avoid umbilic points entirely. The second remark is that the property of being nonumbilic depends only on the jet of the function $\varphi$ of order 4 , and the scalar curvature functions $K_{p, q}^{j}$ depend only on the values (at the origin) of the function $\varphi$ and its derivatives up to order $p+q+2 j$. We may feel free therefore to speak of umbilic or nonumbilic jets (of order at least 4 ) and to view $K_{p, q}^{j}$ as a function on jets (of order at least $p+q+2 j$ ). Finally, the functions $K_{p, q}^{j}$ are real-analytic (in fact polynomial) functions of the jet-variables.

3. The automorphism groups of domains. For $D$ a domain in $\mathbf{C}^{n}$, we write $\operatorname{Aut}(D)$ for the group of biholomorphic automorphisms of $D$. The object of this section is to prove Theorem 2 .

The plan of the proof is to first construct a domain on which $G$ acts by automorphisms, and then to find a strictly pseudoconvex subdomain $D_{0}$ which is $G$-invariant. By using a transversality argument, we then find a perturbation $D$ of $D_{0}$ with the property that points of $b D$ which belong to distinct orbits of $G$ have different Burns-Shnider-Wells curvature invariants. We then show that this boundary rigidity is enough to guarantee that $\operatorname{Aut}(D)=G$.

PROOF. To construct a domain on which $G$ acts by automorphisms, we first use the Peter-Weyl Theorem to imbed $G$ in the complex unitary group $U(n)$ (for some $n)$. We view $U(n)$ as a subgroup of the general linear group $\mathrm{GL}(n)$, which may be identified with a domain in $\mathbf{C}^{n^{2}}$. We then view $G$ as acting on the domain $\mathrm{GL}(n) \times \mathbf{C}^{m}$ in $\mathbf{C}^{n^{2}+m}$ by multiplication in the first factor. (For the moment we leave the integer $m$ unspecified, but it will be chosen later to be very large.) Since matrix multiplication is holomorphic, $G$ acts on $\mathrm{GL}(n) \times \mathbf{C}^{m}$ by automorphisms. It will be convenient to denote points in $\operatorname{GL}(n) \times \mathbf{C}^{m}$ as $(z, w)$ with $z=\left(z_{i j}\right)$ and $w=\left(w_{k}\right)$. This completes the first step.

To construct a $G$-invariant strictly pseudoconvex domain $D_{0}$ in $\operatorname{GL}(n) \times \mathbf{C}^{m}$ which contains $U(n) \times\{0\}$, we consider the real-valued function $\varphi$ defined on $\mathrm{GL}(n) \times \mathbf{C}^{m}$ by

$$
\varphi(z, w)=|\operatorname{det}(z)|^{-2}+\sum\left|z_{i j}\right|^{2}+\sum\left|w_{k}\right|^{2} .
$$

It is easily checked that $\varphi$ is strictly plurisubharmonic and is an exhaustion function for $\mathrm{GL}(n) \times \mathbf{C}^{m}$ in the sense that the sublevel sets $\{\varphi \leq t\}$ are compact for each $t$. We now average $\varphi$ over the group $G$ to obtain $\varphi_{0}$,

$$
\varphi_{0}(z, w)=\int_{G} \varphi(g \cdot z, w) d g
$$


(where $d g$ is the Haar measure on the compact group $G$ ). The function $\varphi_{0}$ is also a strictly plurisubharmonic exhaustion function for $\mathrm{GL}(n) \times \mathbf{C}^{m}$, and is $G$-invariant. Sard's theorem guarantees that most real numbers are regular values of $\varphi_{0}$; since $\varphi_{0}$ is an exhaustion function, we may find a $T$ such that $d \varphi_{0}$ is nonvanishing on $\left\{\varphi_{0}=T\right\}$ and $U(n) \times\{0\} \subset\left\{\varphi_{0}<T\right\}$. Let $D_{0}$ be the connected component of $\left\{\varphi_{0}<T\right\}$ which contains $U(n) \times\{0\}$. Since $U(n) \times\{0\}$ and $\left\{\varphi_{0}<T\right\}$ are invariant under the action of $G$, so is $D_{0}$. Since $T$ is a regular value of $\varphi_{0}$ and $\varphi_{0}$ is a strictly plurisubharmonic function, $D_{0}$ is a strictly pseudoconvex domain. This completes the second step.

The third step is to construct a small, $G$-invariant perturbation $D$ of $D_{0}$ which has the property that points of $b D$ belonging to distinct orbits of $G$ are distinguished by the Burns-Shnider-Wells local holomorphic curvature invariants of $b D$. We will use a transversality argument to construct $D$ as a sublevel set of a $G$ invariant strictly plurisubharmonic function. Note that a $G$-invariant function on a subset of $\mathrm{GL}(n) \times \mathbf{C}^{m}$ is essentially the same thing as a function on a subset of $\left(\mathrm{GL}(n) \times \mathbf{C}^{m}\right) / G$; it is convenient to work in the latter space.

Let $U$ be an open $G$-invariant neighborhood of $b D_{0}$. By choosing $U$ small enough, we may assume that $d \varphi_{0}$ is nonvanishing on $U$. Since $G$ acts by left multiplication in the first factor, the quotient space $U / G$ is a real-analytic manifold which may be identified with an open subset of $(\mathrm{GL}(n) / G) \times \mathbf{C}^{m}$; let $\pi: U \rightarrow U / G$ be the real-analytic quotient map.

Denote by $\mathcal{F}(U / G)$ the family of smooth functions $f: U / G \rightarrow \mathbf{R}$ such that $f \circ \pi$ is strictly plurisubharmonic on $U$ and $d(f \circ \pi)$ is nonvanishing on $U$. Note that $\mathcal{F}(U / G)$ is an open subset of $C^{\infty}(U / G)$ (since strict plurisubharmonicity and nonvanishing of the differential are open conditions) and is not empty (since the $G$-invariant function $\varphi_{0}$ induces on $U / G$ a smooth function which belongs to $\mathcal{F}(U / G)$ ). Note that for $f \in \mathcal{F}(U / G)$, the function $f \circ \pi$ is $G$-invariant on $U$.

For each $r \geq 4$, let $J^{r}(U)$ denote the bundle of jets of order $r$ of real-valued functions on $U$; we write $J^{r}(U)_{x}$ for the fiber of $J^{r}(U)$ at $x$. Let $J_{0}^{r}(U)$ denote the open subset of $J^{r}(U)$ consisting of jets $j_{x}^{r}(\psi)$ such that $\psi$ is strictly plurisubharmonic near $x$ and $(d \psi)_{x} \neq 0$. We let $J^{r}(U / G)$ be the bundle of jets of order $r$ of real-valued functions on $U / G$, and denote by $J_{0}^{r}(U / G)$ the open set of jets $j_{x}^{r}(\psi)$ for which $\psi \circ \pi$ is strictly plurisubharmonic near $\pi^{-1}(x)$ and $d(\psi \circ \pi) \neq 0$ on $\pi^{-1}(x)$. Note that $J^{r}(U)$ and $J^{r}(U / G)$ are real-analytic bundles and that the quotient map $\pi: U \rightarrow U / G$ induces a real-analytic map $\pi_{*}^{r}: J^{r}(U) \rightarrow J^{r}(U / G)$ (see [12, p. 39]), and that $\pi_{*}^{r}\left(J_{0}^{r}(U)\right) \supset J_{0}^{r}(U / G)$.

Let $S_{0}^{r}$ denote the set of umbilic jets in $J_{0}^{r}(U)$ (recall the comment of $\S 2$ ). We will say that a jet $j_{x}^{r}(\psi) \in J_{0}^{r}(U / G)$ is umbilic if $j_{x}^{r}(\psi) \in \pi_{*}^{r}\left(S_{0}^{r}\right)$; this is equivalent to saying that $\psi \circ \pi$ is umbilic at each point of $\pi^{-1}(x)$. (Recall that $G$ acts by holomorphic automorphisms on $U$, hence $\psi \circ \pi$ is umbilic at all points of $\pi^{-1}(x)$ if it is so at one point of $\pi^{-1}(x)$.) Denote the set of umbilic jets in $J_{0}^{r}(U / G)$ by $\Sigma_{0}^{r}$. Since the Burns-Shnider-Wells curvature functions $K_{p, q}^{j}$ depend only on the jet of order $p+q+2 j$ and are biholomorphic invariants (at nonumbilic points) we can also define curvature functions $\tilde{K}_{p, q}$ on $J_{1}^{r}(U / G)=J_{0}^{r}(U / G)-\Sigma_{0}^{r}$ by setting $\tilde{K}_{p, q}\left(j_{x}^{r}(\psi)\right)=K_{p, q}^{0}\left(j_{y}^{r}(\psi \circ \pi)\right)$ for any $y \in \pi^{-1}(x)$, provided that $p+q \leq r, p>q \geq$ 3. (We shall have no use for the other curvature functions $K_{p, q}^{j}$.) Let $S_{p, q}^{r} \subseteq J_{0}^{r}(U)$ 
be the zero set of $K_{p, q}^{0}$ in $J_{0}^{r}(U)-S_{0}^{r}$ and let $\Sigma_{p, q}^{r}$ be the zero set of $\tilde{K}_{p, q}$ in $J_{0}^{r}(U / G)-\Sigma_{0}^{r}$.

Since we are going to use a transversality argument in $J_{0}^{r}(U / G)$, we need some information about the sets $\Sigma_{0}^{r}$ and $\Sigma_{p, q}^{r}$. In [6] it is shown that each of the sets $S_{0}^{r}$ and $S_{p, q}^{r}$ is a smooth submanifold, and the exact codimensions are calculated. Such precise information is not readily available for the sets $\Sigma_{0}^{r}$ and $\Sigma_{p, q}^{r}$; fortunately, we can do with less. What we need, in essence, is to be sure that the jets $j^{r}(f)$ of most functions $f \in \mathcal{F}(U / G)$ miss the sets $\Sigma_{0}^{r}$ and $\Sigma_{p, q}^{r}$.

It will help to recall two facts from real-analytic geometry: (A) every realanalytic variety is the union of countably many real-analytic manifolds; (B) the image of a real-analytic variety under a real-analytic mapping is the union of countably many real-analytic manifolds. (Fact (A) is due to Whitney; see [19] for example. Fact (B) is well known, but since there seems to be no convenient reference, we give a sketch. Note that the assertion is local and that by fact (A) it is enough to consider real-analytic manifolds, so let $f: \mathbf{R}^{s} \rightarrow \mathbf{R}^{t}$ be real-analytic and let $\mathbf{X}=\left\{x \in \mathbf{R}^{s}: \operatorname{rank}(d f)_{x}=t\right\}$. Then $\mathbf{X}$ is open and $f(\mathbf{X})$ is open in $\mathbf{R}^{t}$. The set $\mathbf{R}^{s}-\mathbf{X}$ is a real-analytic variety and hence is the union of manifolds; let $\mathbf{X}^{\prime}$ be any of these manifolds and choose a point $x \in \mathbf{X}^{\prime}$ where $\left(d\left(f_{\mid \mathbf{X}^{\prime}}\right)\right)_{x}$ has maximal rank-say $r<t$. In a neighborhood $Y$ of $x$ in $\mathbf{X}^{\prime}, f_{\mid \mathbf{X}^{\prime}}$ may be written as a function of $r$ of the coordinates, so the image $f(Y)$ will be an $r$-dimensional submanifold of $\mathbf{R}^{t}$. Continuing downward in this fashion yields the result.)

We now look at $\Sigma_{0}^{r}$; we want to show that it is contained in the countable union of real-analytic submanifolds of high codimension, provided that $m$ and $r$ are chosen appropriately. Since $\Sigma_{0}^{r}$ is contained in $\pi_{*}^{r}\left(S_{0}^{r}\right)$, we need only prove that $\pi_{*}^{r}\left(S_{0}^{r}\right)$ is contained in the countable union of submanifolds of high codimension. Since a jet in $J_{0}^{r}(U / G)$ lies in $\pi_{*}^{r}\left(S_{0}^{r}\right)$ if and only if its truncation in $J_{0}^{4}(U / G)$ lies in $\pi_{*}^{4}\left(S_{0}^{4}\right)$ (because the property of being umbilic depends only on jets of order 4), it suffices to consider $\pi_{*}^{4}\left(S_{0}^{4}\right)$. Now, it is shown in $[\mathbf{6}]$ that $S_{0}^{4}$ is a real-analytic subvariety of $J_{0}^{4}(U)$; by fact (B) above, $\pi_{0}^{4}\left(S_{0}^{4}\right)$ is a countable union of submanifolds of $J_{0}^{4}(U / G)$. Moreover in [6] the codimension of $S_{0}^{4}$ in $J_{0}^{4}(U)$ is shown to be $\frac{1}{4}(t-1)^{2} t^{2}-(t-1)^{2}$, where $t=n^{2}+m=\operatorname{dim}_{\mathbf{C}}(U)$. Thus the codimension of $\pi_{*}^{4}\left(S_{0}^{4}\right)$ in $J_{0}^{4}(U / G)$ (i.e., the minimal codimension of a submanifold of $\left.\pi_{*}^{4}\left(S_{0}^{4}\right)\right)$ is at least

$$
\frac{1}{4}\left[(t-1)^{2} t^{2}-(t-1)^{2}\right]-\left[\operatorname{dim} J^{4}(U)-\operatorname{dim} J^{4}(U / G)\right] .
$$

For our purposes it is enough to note that (since $t=n^{2}+m$ ) this codimension is of the form $\frac{1}{4} m^{4}-A_{0}$ where $A_{0}$ is an expression in $n, m$ and $\operatorname{dim} G$ which involves, no terms in $m$ of higher order than $m^{3}$. Since $n$ and $\operatorname{dim} G$ are fixed and we are free to choose $m$ as large as we like, we can guarantee that the codimension of $\pi_{*}^{4}\left(S_{0}^{4}\right)$ in $J_{0}^{4}(U / G)$ is at least $m^{3}$. In view of our previous remark this means that the codimension of $\pi_{*}^{r}\left(S_{0}^{r}\right)$ in $J_{0}^{r}(U / G)$ is at least $m^{3}$ (if $m$ is large enough), and this estimate is independent of $r$.

We now use the same trick on $\Sigma_{p, q}^{r}$ (for $p>q \geq 3$ and $p+q \leq r$ ). Since $\Sigma_{p, q}^{r}$ is the zero set of a real-analytic function, it is a real-analytic subvariety of $J_{1}^{r}(U / G)$. Moreover, $S_{p, q}^{r}$ is a smooth real-analytic subvariety of $J_{1}^{r}(U)=J_{0}^{r}(U)-S_{0}^{r}$ of codimension

$$
2\left(\begin{array}{c}
t+p-2 \\
t-2
\end{array}\right)\left(\begin{array}{c}
t+q-2 \\
t-2
\end{array}\right)
$$


(see [6]), and $\pi_{*}^{r}\left(S_{p, q}^{r}\right) \supset \Sigma_{p, q}^{r}$. As before, we may calculate the codimension of $\pi_{*}^{p+q}\left(S_{p, q}^{p+q}\right)$ in $J_{1}^{p+q}(U / G)$ and conclude that this is at least the codimension of $\Sigma_{p, q}^{r}$ in $J_{1}^{r}(U / G)$, since vanishing of $K_{p, q}^{0}$ depends only on jets of order $p+q$. A little care must be exercised here, but it may be seen that: if $m$ is sufficiently large then the codimension of $\Sigma_{p, q}^{r}$ in $J_{1}^{r}(U / G)$ is at least $m^{3}$ whenever $p>q \geq 3$. (There is nothing special about $m^{3}$ here; we could do better by several powers of $m$, but this agrees with the estimate for $\Sigma_{0}^{r}$, and is more than adequate for our purposes.) We will also need to take $m^{3}>2\left(n^{2}+m\right)$.

We now fix $m$ sufficiently large (as above) and write $\Sigma=\Sigma_{0}^{m} \cup \cup \Sigma_{p, q}^{m}$, where the second union extends over all $p, q$ with $p>q \geq 3, p+q \leq m$. By the above, $\Sigma$ is a countable union of real-analytic submanifolds of $J_{0}^{m}(U / G)$ each of which has codimension at least $m^{3}$. Note that the real dimension $U / G$ is at most $2 t=2\left(n^{2}+m\right)$, so Thom's jet transversality theorem (see [12]) guarantees that there is a dense $G_{\delta}$-set $E$ in $\mathcal{F}(U / G)$ such that for each $f \in E$ the jet $j^{m}(f)$ is transversal to each submanifold of $\Sigma$. In these dimensions, this means that $j^{m}(f)$ misses $\Sigma$ entirely.

We let $J_{2}^{m}(U / G)=J_{1}^{m}(U / G)-\Sigma$. By letting $m$ be sufficiently large, we can choose $2 t+1=2\left(n^{2}+m\right)+1$ distinct curvature functions $\tilde{\mathbf{K}}_{1}, \ldots, \tilde{\mathbf{K}}_{2 t+1}$, where each $\tilde{\mathbf{K}}_{s}=\tilde{\mathbf{K}}_{p_{s}, q_{s}}$ for $p_{s}>q_{s} \geq 3, p_{s}+q_{s} \leq m$, such that

$$
\tilde{\mathbf{K}}=\left(\tilde{\mathbf{K}}_{1}, \ldots, \tilde{\mathbf{K}}_{2 t+1}\right): J_{2}^{m}(U / G) \rightarrow \mathbf{R}^{2 t+1}
$$

has maximal rank (since some $K=\left(K_{1}, \ldots, K_{2 t+1}\right)$ has maximal rank in each fiber when only the $\mathbf{C}^{m}$-variables are considered; see [6]). Let $\Delta$ be the diagonal of $\mathbf{R}^{2 t+1} \times \mathbf{R}^{2 t+1} ;$ then $(\tilde{\mathbf{K}}, \tilde{\mathbf{K}})^{-1}(\Delta)$ is a submanifold of $J_{2}^{m}(U / G)$ of codimension $2 t+$ 1. Mather's multijet transversality theorem then implies that there is a dense $G_{\delta}$-set $E^{\prime}$ in $\mathcal{F}(U / G)$ such that for each $f \in E^{\prime}$, the jet $j^{m}(f)$ misses $\Sigma$, and $\left(j^{m}(f), j^{m}(f)\right)$ is transversal to $(\tilde{\mathbf{K}}, \tilde{\mathbf{K}})^{-1}(\Delta)$ (intersections computed in $J_{0}^{r}(U / G) \times J_{0}^{r}(U / G)$ ). In these dimensions, this means that $\left(j_{x}^{m}(f), j_{y}^{m}(f)\right)$ is not in $(\tilde{\mathbf{K}}, \tilde{\mathbf{K}})^{-1}(\Delta)$ if $x \neq y$.

Define the function $f_{0} \in \mathcal{F}(U / G)$ by $f_{0} \circ \pi=\varphi_{0}$; by the above, we can find a function $f \in \mathcal{F}(U / G)$ arbitrarily close to $f_{0}$ such that $j^{m}(f)$ misses $\Sigma$ and $\left(j^{m}(f), j^{m}(f)\right)$ is transversal to $(\tilde{\mathbf{K}}, \tilde{\mathbf{K}})^{-1}(\Delta)$. Set $\varphi=f \circ \pi$ and let $x_{0} \in b D_{0}$; then the level set $\left\{x \in U: \varphi(x)=\varphi\left(x_{0}\right)\right\}$ is a hypersurface close to $b D_{0}$, so this level set bounds a domain $D$ in $\operatorname{GL}(n) \times \mathbf{C}^{m}$. Since $\varphi$ is close to $\varphi_{0}$, this domain $D$ is strictly pseudoconvex. Since $\varphi=f \circ \pi$ is a $G$-invariant function, $D$ is a $G$-invariant set. Moreover, for each pair of points $x, y \in b D$ with $y \notin G \cdot x$, our construction guarantees that the scalar curvature invariants $K_{1}, \ldots, K_{2 t+1}$ do not all agree at $x$ and $y$. This completes the third step.

The fourth step is simply to observe that if $h$ is a holomorphic automorphism of $D$ then the extension $\hat{\mathbf{h}}$ of $h$ to $\overline{\mathbf{D}}$ preserves the holomorphic curvature invariants. Hence if $x, y \in b D$ and $y \notin G \cdot x$, it cannot be the case that $\hat{\mathbf{h}}(x)=y$. Equivalently, for every automorphism $h \in \operatorname{Aut}(D)$, the mapping $\hat{\mathbf{h}}: \overline{\mathbf{D}} \rightarrow \overline{\mathbf{D}}$ leaves invariant all the orbits of $G$ which lie in $b D$, and hence leaves invariant all the $G$-invariant subsets of $b D$.

The fifth step is to show that all the automorphisms of $D$ leave invariant all the orbits of $G$ inside $D$; i.e., for $x \in D, h \in \operatorname{Aut}(D)$ there is a (unique) $g \in G$ with $h(x)=g \cdot x$. To do this, we need some information about the way $U(n)$ and $G$ sit in $\mathrm{GL}(n)$. 
Let us view elements of $\mathrm{GL}(n)$ as linear transformations on $\mathbf{C}^{n}$, and equip $\mathbf{C}^{n}$ with its usual inner product. Then a matrix $y \in \operatorname{GL}(n)$ is unitary if and only if $\|z\|=\left\|z^{-1}\right\|=1$, where $\|z\|$ is the norm of $z$ as a linear transformation; i.e.,

$$
\|z\|=\sup \left\{|\langle z \sigma, \tau\rangle|: \sigma, \tau \in \mathbf{C}^{n},\|\sigma\|=\|\tau\|=1\right\} .
$$

Hence for each $z_{0} \in \mathrm{GL}(n)$ which does not belong to $U(n)$, there are vectors $\sigma, \tau \in \mathbf{C}^{n}$ with $\|\sigma\|=\|\tau\|=1$ such that either $\left|\left\langle z_{0} \sigma, \tau\right\rangle\right|>1$ or $\left|\left\langle z_{0}^{-1} \sigma, \tau\right\rangle\right|>1$. Because the maps $z \rightarrow z^{-1}$ and $z \rightarrow\langle z \sigma, \tau\rangle$ are holomorphic on $\operatorname{GL}(n)$, we conclude in either case there is a holomorphic function $F$ on $\operatorname{GL}(n)$ such that $F\left(z_{0}\right)>$ 1 , while $|F(z)| \leq 1$ for each $z \in U(n)$. It now follows from a result of Björk [4, Theorem 6.1] that every continuous complex-valued function on $U(n)$ can be uniformly approximated by restrictions to $U(n)$ of holomorphic functions on $\operatorname{GL}(n)$. As a consequence, it follows that for each $z_{0} \in \mathrm{GL}(n)$ which does not belong to $G$, there is a holomorphic function $F$ on $\mathrm{GL}(n)$ such that $F\left(z_{0}\right)>1$ while $|F(g)| \leq 1$ for each $g \in G$. (If $z_{0} \notin U(n)$, we have constructed such a function above; if $z_{0} \in U(n)$, we simply find a continuous function on $U(n)$ with the desired property and then approximate it sufficiently well by a holomorphic function on $\operatorname{GL}(n)$.)

Now let $h$ be an automorphism of $D$, let $\left(z_{0}, w_{0}\right) \in D$, and write $h\left(z_{0}, w_{0}\right)=$ $\left(z^{*}, w^{*}\right)$; we want to show that there is a unique element $g \in G$ such that $g$. $\left(z_{0}, w_{0}\right)=\left(g \cdot z_{0}, w_{0}\right)=\left(z^{*}, w^{*}\right)$. To do this, we first show that $z^{*}=g \cdot z_{0}$ (for some $g \in G$ ). If this were not so, we could choose-by the above-a holomorphic function $F$ on $\operatorname{GL}(n)$ such that $F\left(z^{*} z_{0}^{-1}\right)>1$ and $|F(g)| \leq 1$ for each $g \in G$. Define a holomorphic function $F_{1}$ on $\operatorname{GL}(n) \times \mathbf{C}^{m}$ by $F_{1}(z, w)=F\left(z z_{0}^{-1}\right)$, so that $F_{1}\left(z^{*}, w^{*}\right)>1$ and $\left|F_{1}\left(g \cdot z_{0}, w\right)\right| \leq 1$ for each $g \in G, w \in \mathbf{C}^{m}$. Set

$$
\mathbf{X}=\left\{\left(g \cdot z_{0}, w\right) \in \mathrm{GL}(n) \times \mathbf{C}^{m}: g \in G, w \in \mathbf{C}^{m}\right\},
$$

so that $\left|F_{1}\right| \leq 1$ on $\mathbf{X}$. Write $\hat{\mathbf{h}}$ for the smooth extension of $h$ to the closure $\overline{\mathbf{D}}$ of $D$, and set $\mathbf{Y}=\hat{\mathbf{h}}(\mathbf{X} \cap \overline{\mathbf{D}})$. Evidently, $\mathbf{X} \cap \overline{\mathbf{D}}$ is the union of complex submanifolds with boundary of $D$, so $\mathbf{Y}$ is also the union of such submanifolds with boundary. The maximum principle for holomorphic functions implies that $\left|F_{1}(y)\right| \leq \sup \left\{\left|F_{1}\left(y^{\prime}\right)\right|: y^{\prime} \in \mathbf{Y} \cap b D\right\}$ for each $y \in \mathbf{Y}$. On the other hand, $\mathbf{X} \cap b D$ is obviously a $G$-invariant subset of $b D$; hence (by the previous step) we conclude that $\mathbf{Y} \cap b D=\hat{\mathbf{h}}(\mathbf{X} \cap b D)=\mathbf{X} \cap b D$, which implies $\left|F_{1}(y)\right| \leq 1$ for each $y \in \mathbf{Y}$. In particular, $\left|F_{1}\left(z^{*}, w^{*}\right)\right|=\left|F_{1}\left(h\left(z_{0}, w_{0}\right)\right)\right| \leq 1$ which is a contradiction. We conclude that $z^{*}=g \cdot z_{0}$ for some $g \in G$, as asserted.

Next we show that $w^{*}=w_{0}$. To see this, we simply note that $\mathbf{V}=\{(z, w) \in \overline{\mathbf{D}}$ : $\left.w=w_{0}\right\}$ is a complex submanifold with boundary of $D$ whose boundary lies in $b D$ and is $G$-invariant. Hence $\hat{\mathbf{h}}(\mathbf{V})$ is also a submanifold with boundary of $\overline{\mathbf{D}}$ and $\hat{\mathbf{h}}(\mathbf{V}) \cap b D=\mathbf{V} \cap b D$. As before, this means that

$$
|f(y)| \leq \sup \left\{\left|f\left(y^{\prime}\right)\right|: y^{\prime} \in \hat{\mathbf{h}}(\mathbf{V}) \cap b D=\mathbf{V} \cap b D\right\}
$$

for each holomorphic function $f$ on $\operatorname{GL}(n) \times \mathbf{C}^{m}$. Since the functions $(z, w) \rightarrow$ $w_{k}-\left(w_{0}\right)_{k}$ are holomorphic on $\operatorname{GL}(n) \times \mathbf{C}^{m}$ and vanish on $\mathbf{V}$, we conclude that they also vanish on $\hat{\mathbf{h}}(\mathbf{V})$. Since $\left(z^{*}, w^{*}\right) \in \hat{\mathbf{h}}(\mathbf{V})$, we conclude that $\left(w^{*}\right)_{k}=\left(w_{0}\right)_{k}$ for each $k$; i.e., $w^{*}=w_{0}$, as asserted. Thus, $\left(z^{*}, w^{*}\right)=g \cdot\left(z_{0}, w_{0}\right)=\left(g \cdot z_{0}, w_{0}\right)$ for some $g \in G$. 
It remains to show that $g$ is unique; this is clearly the case since $G$ acts by multiplication in the first factor. This completes the fifth step.

The final step is to show that $\operatorname{Aut}(D)=G$; i.e., for each $h \in \operatorname{Aut}(D)$, there is a $g \in G$ with $h(z, w)=g \cdot(z, w)$ for each $(z, w) \in D$. To see this, fix an automorphism $h$. For each $(z, w) \in D$, let $g(z, w)$ be the unique element of $G$-constructed in the previous step-for which $h(z, w)=g(z, w) \cdot(z, w)=(g(z, w) \cdot z, w)$. We may then write $h(z, w)=\left(h_{1}(z, w), w\right)$, where $h_{1}$ is a holomorphic function from $D$ into $\mathrm{GL}(n)$. Multiplying on the right by $z^{-1}$ yields $g(z, w)=h_{1}(z, w) \cdot z^{-1}$ for each $(z, w) \in D$. Hence the map $\tau: D \rightarrow \operatorname{GL}(n)$ which sends the point $(z, w)$ to the group element $g(z, w)$ is a holomorphic map from $D$ into $\operatorname{GL}(n)$ whose range is actually contained in $G$. Consequently, the differential $d \tau$ of this map sends the tangent bundle of $D$ into the tangent bundle of $G$ (viewed as a subbundle of the tangent bundle of GL $(n)$ ). On the other hand, the differential of a holomorphic map is complex-linear on each fiber of the tangent bundle. Hence $(d \tau)_{(z, w)}\left(T_{(z, w)} D\right)$ must be a complex subspace of $T_{g(z, w)} G$ (which is a subspace of $T_{g(z, w)} \operatorname{GL}(n)$ ), for each $(z, w) \in D$. As is easily seen, however, the unitary group $U(n)$ has no nonzero complex tangent vectors in $\operatorname{GL}(n)$; in particular $T_{g(z, w)} G$ contains no complex subspaces (of positive dimension) of $T_{g(z, w)} \mathrm{GL}(n)$. It follows that $(d \tau)_{(z, w)}=0$ for each $(z, w) \in D$; since $D$ is connected, we conclude that $\tau$ is a constant mapping. In other words, there is an element $g \in G$ such that $h(z, w)=g \cdot(z, w)$ for all $(z, w) \in D$. This completes the final step, and with it, the proof of Theorem 2 .

It is worth noting that the domain $D$ can be chosen to have real-analytic boundary. This is an immediate corollary of a result of Greene and Krantz [14], who have shown that for each strictly pseudoconvex domain $D$ there is a subdomain $D^{\prime}$ with real-analytic boundary, such that $D^{\prime}$ is invariant under $\operatorname{Aut}(D)$ and $\operatorname{Aut}\left(D^{\prime}\right)=$ $\operatorname{Aut}(D)$.

Notice that when we view $\operatorname{GL}(n) \times \mathbf{C}^{m}$ as a domain in $\mathbf{C}^{n^{2}+m}$, the group $G$ acts on the domain $D$ by linear transformations of the ambient space, and of course $G$ acts freely on $D$. By using a ball in $\mathbf{C}^{n^{2}+m}$ as our basic domain $D_{0}$-instead of a small neighborhood of $U(N) \times\{0\}$ in $\operatorname{GL}(n) \times \mathbf{C}^{m}$-we could choose $D$ so that the group $G$ has a fixed point in $D$ (and again acts by linear transformations of the ambient space). It does seem to be important for our argument, however, that $G$ act freely on some relatively open $G$-invariant subset of $b D$.

Before turning our attention to isometries, it seems appropriate to point out that the holomorphic nature of the automorphisms is crucial in the last step, where the fact that an automorphism $h$ leaves invariant all orbits of $G$ in $D$ guarantees that $h$ actually belongs to $G$. As noted in the introduction, we cannot a priori draw the same conclusion for isometries of a Riemannian manifold. In effect, the way we use Theorem 2 in the proof of Theorem 1 will be to construct a setting in which isometries not belonging to $G$ actually do move some $G$-orbit.

4. The Bergman metric, antiholomorphic automorphisms, and perturbations of Riemannian metrics. In order to apply Theorem 2 to the problem of isometry groups of Riemannian manifolds, we need some detailed information about the Bergman metric of a strictly pseudoconvex domain. We also need a result of Ebin about the behavior of isometry groups under perturbations of a metric. This section collects the information we need. 
Let $D$ be a strictly pseudoconvex domain in $\mathbf{C}^{n}$ and let $V$ be the ordinary Euclidean volume on $D$. We denote by $L^{2}(D, V)$ the Hilbert space of squareintegrable complex-valued functions on $D$, and by $H^{2}(D, V)$ the closed subspace of $L^{2}(D, V)$ consisting of holomorphic functions; write $P: L^{2}(D, V) \rightarrow H^{2}(D, V)$ for the orthogonal projection. The Bergman kernel $B_{D}(\cdot, \cdot)$ is the integration kernel which represents $P$; i.e.,

$$
\operatorname{Pf}(z)=\int_{D} f(w) B_{D}(z, w) d V(w)
$$

for each $f \in L^{2}(D, V), z \in D$. The function $B_{D}(\cdot, w)$ is in $H^{2}(D, V)$ (for each $w \in D$ ) and $\bar{B}_{D}(z, \cdot)$ is in $H^{2}(D, V)$ (for each $z \in D$ ). Moreover, $B_{D}$ is a smooth function on $D \times D$. (For more information about the Bergman kernel we refer to [25].) We write $\tilde{\mathbf{B}}_{D}(z)=B_{D}(z, z)$.

To define the Bergman metric, we consider the Levi form of the function $\tilde{\mathbf{B}}_{D}$ :

$$
L\left(\log \tilde{\mathbf{B}}_{D}\right)=\sum \frac{\partial^{2}\left(\log \tilde{\mathbf{B}}_{D}\right)}{\partial z_{i} \partial \bar{z}_{j}} d z_{i} \wedge d \bar{z}_{j}
$$

This 2-form is the imaginary part of a unique smooth Hermitian metric $\beta_{D}^{*}$ on the complex tangent bundle of $D$. If we view $\beta_{D}^{*}$ as acting on the real tangent bundle of $D$, and take its real part, we obtain a smooth Riemannian metric $\beta_{D}$, the Bergman metric of $D$.

We will make use of the following facts about the Bergman metric $\beta_{D}$ of a strictly pseudoconvex domain $D$ with smooth boundary.

$$
\begin{aligned}
& \beta_{D} \text { is a complete metric and is invariant under biholomorphic map- } \\
& \text { pings of } D[\mathbf{2 5}] \text {. }
\end{aligned}
$$

If $\kappa(\beta, x)$ denotes the scalar curvature of the metric $\beta$ at the point $x$, and $\beta_{0}$ is the Bergman metric of the unit ball in $\mathbf{C}^{n}$, then $\kappa\left(\beta_{0}, x\right)=\kappa\left(\beta_{0}, 0\right)$ for each $x$ in the ball.

For any strictly pseudoconvex domain $D$, the scalar curvature $\kappa\left(\beta_{D}, x\right)$ approaches $\kappa\left(\beta_{0}, 0\right)$ uniformly as $x$ approaches $b D[\mathbf{1 6}$, 13].

If $D$ is not biholomorphically equivalent to the ball then there is at least one point $x \in D$ where $\kappa\left(\beta_{D}, x\right) \neq \kappa\left(\beta_{0}, 0\right)[14]$.

We will write $\operatorname{Aut}^{*}(D)$ for the (possibly empty) set of antiholomorphic automorphisms of $D$, and set

$$
\operatorname{Aut}_{0}(D)=\operatorname{Aut}(D) \cup \operatorname{Aut}^{*}(D) .
$$

It is easily checked that $\operatorname{Aut}_{0}(D)$ is a group and that $\operatorname{Aut}(D)$ is a normal subgroup of $\operatorname{Aut}_{0}(D)$ which is of index 2 if $\operatorname{Aut}^{*}(D) \neq \varnothing$. We note that every element of $\operatorname{Aut}^{*}(D)$ extends smoothly to $\overline{\mathbf{D}}$ (since $J(D)$ is a strictly pseudoconvex domain, so that Fefferman's theorem may be applied to $J \circ f: D \rightarrow J(D))$. The relevance of $\operatorname{Aut}_{0}(D)$ to the Bergman metric is

the isometry goup of $D$ with respect to the Bergman metric $\beta_{D}$ is precisely $\operatorname{Aut}_{0}(D)[\mathbf{1 4}]$. 
Finally we need some information about perturbations of metrics. Let $M$ be a compact, smooth Riemannian manifold (without boundary) and let $\gamma_{0}$ be a smooth Riemannian metric on $M$. The following theorem of Ebin [10] details the variation of the isometry group with the variation of the metric $\gamma_{0}$.

THEOREM. Let $\left\{\gamma_{k}: k=1,2, \ldots\right\}$ be a sequence of smooth Riemannian metrics on $M$ converging (in the $C^{\infty}$-sense of convergence of metrics) to $\gamma_{0}$. Then there is a sequence $\left\{F_{k}: k=k_{0}, k_{0}+1, \ldots\right\}$ of diffeomorphisms of $M$ which converges (in the $C^{\infty}$-sense) to the identity such that if $f: M \rightarrow M$ is an isometry of $\gamma_{k}$ then $F_{k} \circ f \circ F_{k}: M \rightarrow M$ is an isometry of $\gamma_{0}\left(\right.$ for $\left.k \geq k_{0}\right) ;$ i.e., $F_{k}$ conjugates the isometry group of $\gamma_{k}$ to a subgroup of the isometry group of $\gamma_{0}$.

5. The isometry groups of manifolds. If $M$ is a smooth manifold and $\gamma$ is a smooth Riemannian metric on $M$, we write $\operatorname{Isom}(M, \gamma)$ for the group of diffeomorphisms of $M$ which are isometries of the metric $\gamma$. The object of this section is to prove Theorem 1 .

The plan of the proof is to use the domain $D$ constructed in Theorem 2 and construct a metric $\mu$ on $\overline{\mathbf{D}}$ which is straight at the boundary and whose isometries are precisely the holomorphic and antiholomorphic automorphisms of $D$. The manifold $M$ will be the double of $\overline{\mathbf{D}}$, and the metric $\gamma$ will be obtained by perturbing the double of the metric $\mu$ on $\overline{\mathbf{D}}$.

PROOF. Theorem 2 guarantees that we can find a strictly pseudoconvex domain $D$ in $\operatorname{GL}(n) \times \mathbf{C}^{m} \subset \mathbf{C}^{n^{2}+m}$ which contains $U(n) \times\{0\}$, on which $G$ acts by left multiplication in the first factor and for which $\operatorname{Aut}(D)=G$.

The next step is to construct a metric $\mu$ on $\overline{\mathbf{D}}$ which is a product metric near $b D$ and has the property that

$$
\operatorname{Isom}(\overline{\mathbf{D}}, \mu)=\operatorname{Aut}_{0}(D)=\operatorname{Isom}\left(D, \beta_{D}\right) .
$$

This is essentially Proposition 21 of [14]. Unfortunately, the argument given there has a gap; a sketch of a correction is given in [15]. We give a complete argument, following the lines of $[\mathbf{1 4}, \mathbf{1 5}]$.

We first choose a smooth metric $\alpha^{\prime}$ on $D$ which is $\operatorname{Aut}_{0}(D)$-invariant. (Begin with an arbitrary metric on $D$ and average it over the compact group $\operatorname{Aut}_{0}(D)$.) Let $d$ be the distance function on $D$ induced by $\alpha^{\prime}$. If $\lambda>0$ is sufficiently small, then $A=\{x \in \overline{\mathbf{D}}: d(x, b D)<\lambda\}$ is an $\operatorname{Aut}_{0}(D)$-tubular neighborhood of $b D$; i.e., $A=b D \times[0, \lambda)$ with $\operatorname{Aut}_{0}(D)$ acting on the first factor. Let $\alpha$ be any metric on $b D$ which is invariant for $\operatorname{Aut}_{0}(D)$, and let $\alpha^{\prime \prime}$ be the metric on $A$ which is the product of $\alpha$ on $b D$ and the Euclidean metric on $[0, \lambda)$. (For the moment, we leave the metric $\alpha$ unspecified; in the last step of the proof, we construct a specific metric.)

Write $\beta$ for the Bergman metric of $D$, and for each $x \in D$, write $\kappa(x)$ for the scalar curvature of $\beta$ at $x$; let $\kappa_{0}$ be the scalar curvature of the Bergman metric of the unit ball in $\mathbf{C}^{n^{2}+m}$. By construction, the domain $D$ is not biholomorphically equivalent to the ball (because $\operatorname{Aut}(D)=G$ is compact), so (see $\S 4$ ), there is at least one point $x_{0} \in D$ for which $\kappa(x) \neq \kappa_{0}$. On the other hand, $\kappa(x)$ tends to $\kappa_{0}$ as $x$ tends to $b D$. Hence we can find a real number $s>0$ such that $K=\left\{x \in D:\left|\kappa(x)-\kappa_{0}\right| \geq s\right\}$ is a compact subset of $D$ with nonempty interior.

Let $d_{\beta}$ denote the distance function on $D$ induced by $\beta$. For each $\rho>0$, set $D_{\rho}=\left\{x \in D: d_{\beta}(x, K)<\rho\right\}$. Since $\beta$ is a complete metric, each $D_{\rho}$ is 
a relatively compact, open subset of $D$ containing $K$. Moreover, $D_{\rho_{1}} \subset D_{\rho_{2}}$ if $\rho_{1}<\rho_{2}$ and $\bigcup_{\rho \geq 0} D_{\rho}=D$. Since $A$ is a neighborhood of $b D$ we can find a $\rho_{0}$ so large that $D_{\rho_{0}} \cup A \supset D$. We may also choose $\rho_{0}$ sufficiently large to have $\operatorname{vol}_{\beta}(K)>2 \operatorname{vol}_{\alpha^{\prime \prime}}\left(D \backslash D_{\rho_{0}}\right)$ (where $\operatorname{vol}_{\beta}$ and $\operatorname{vol}_{\alpha^{\prime \prime}}$ denote volumes with respect to the metrics $\beta$ and $\alpha^{\prime \prime}$ respectively). Finally, we may also choose $\rho_{0}$ to be larger than the diameter of $K$ in the distance $d_{\beta}$.

For each $\rho \geq \rho_{0}$, choose a smooth, $\operatorname{Aut}_{0}(D)$-invariant function $\psi: \overline{\mathbf{D}} \rightarrow \mathbf{R}$ which is equal to 1 on $D_{\rho}$ and equal to 0 on a neighborhood of $b D$ contained in $A$. Define a smooth metric $\mu(\rho)$ on $\overline{\mathbf{D}}$ by $\mu(\rho)=\psi \beta+(1-\psi) \alpha^{\prime \prime}$. Notice that since $K$ is defined by curvature inequalities for $\beta$, and since $D_{\rho}$ is defined by distance inequalities for $\beta$, and $\operatorname{Aut}_{0}(D)=\operatorname{Isom}(D, \beta)$, both $K$ and $D_{\rho}$ are $\operatorname{Aut}_{0}(D)$-invariant. Hence we may choose $\psi$ to be supported in a neighborhood of $D_{\rho}$ which is so small that $\operatorname{vol}_{\mu(\rho)}\left(D \backslash D_{\rho}\right)$ is nearly $\operatorname{vol}_{\alpha^{\prime \prime}}\left(D \backslash D_{\rho}\right)$. In particular, we can arrange that

$$
\operatorname{vol}_{\mu(\rho)}(K)=\operatorname{vol}_{\beta}(K)>2 \operatorname{vol}_{\mu(\rho)}\left(D \backslash D_{\rho}\right) .
$$

We want to show that $\operatorname{Isom}(\overline{\mathbf{D}}, \mu(\rho))=\operatorname{Aut}_{0}(D)$, provided that $\rho$ is sufficiently large. Note first of all that

$$
\operatorname{Aut}_{0}(D)=\operatorname{Isom}(D, \beta) \subset \operatorname{Isom}(\overline{\mathbf{D}}, \mu(\rho))
$$

for every $\rho \geq \rho_{0}$, since $\beta, \alpha^{\prime \prime}$, and $\psi$ are all $\operatorname{Aut}_{0}(D)$-invariant. To obtain the reverse inclusion, we want to compare $\operatorname{Aut}_{0}(D), \operatorname{Isom}(\overline{\mathbf{D}}, \mu(\rho))$, and $\operatorname{Isom}\left(D_{\rho}, \beta\right)$; of course these groups act on different spaces, but we can compare them if we can find a common invariant set.

In fact we assert that $K$ is invariant for each of these groups. It is clear that $K$ is invariant for $\operatorname{Aut}_{0}(D)=\operatorname{Isom}(D, \beta)$ and for $\operatorname{Isom}\left(D_{\rho}, \beta\right)$, since $K$ is defined by curvature inequalities of $\beta$. To see that $K$ is also invariant for $\operatorname{Isom}(\overline{\mathbf{D}}, \mu(\rho))$, let $f \in \operatorname{Isom}(\overline{\mathbf{D}}, \mu(\rho))$, and consider the image $f(K)$. Because $K$ is defined by curvature inqualities of $\beta$ and $\mu(\rho)=\beta$ on $D_{\rho}$, it is certainly the case that $f(K) \cap\left(D_{\rho} \backslash K\right)=\varnothing$. Since isometries preserve volume and $\operatorname{vol}_{\mu(\rho)}(K)>2 \operatorname{vol}_{\mu(\rho)}\left(D \backslash D_{\rho}\right)$, it is also the case that $f(K)$ is not contained in $D \backslash D_{\rho}$. Since $D_{\rho}$ is a neighborhood of $K$ on which $\mu(\rho)=\beta$, it is also the case that $d_{\mu(\rho)}(y, K) \geq \rho \geq \rho_{0}$ for all $y \in D \backslash D_{\rho}$. Since isometries preserve distances, and hence diameters of sets, we see (recalling that the diameter of $K$ for the distance function $d_{\beta}$ is less than $\left.\rho_{0}\right)$ that $f(K)$ cannot meet both $K$ and $D \backslash D_{\rho}$. Putting all this information together yields that $f(K) \subset K$, i.e., $K$ is invariant for $\operatorname{Isom}(\overline{\mathbf{D}}, \mu(\rho))$.

If we restrict to the invariant set $K$, we obtain the following inclusions:

$$
\left.\left.\left.\operatorname{Aut}_{0}(D)\right|_{K} \subset \operatorname{Isom}(\overline{\mathbf{D}}, \mu(\rho))\right|_{K} \subset \operatorname{Isom}\left(D_{\rho}, \beta\right)\right|_{K}
$$

whenever $\rho \geq \rho_{0}$. The first inclusion follows from the fact that $\mu(\rho)$ is $\operatorname{Aut}_{0}(D)$ invariant. The second inclusion follows from the facts that $\mu(\rho)=\beta$ on $D_{\rho}$ and that $D_{\rho}$ is invariant for $\operatorname{Isom}(\overline{\mathbf{D}}, \mu(\rho))$ and $\operatorname{Isom}\left(D_{\rho}, b\right)$ (since it is defined by a distance inequality involving $\beta$ and the invariant set $K)$. Note that $\left.\operatorname{Isom}\left(D_{\rho_{1}}, \beta\right)\right|_{K} \supset$ $\left.\operatorname{Isom}\left(D_{\rho_{2}}, \beta\right)\right|_{K}$ whenever $\rho_{1} \leq \rho_{2}$, and that each of these groups is compact. (This is because the orbit of any point in $K$ lies in $K$-which is compact-and because the isotropy subgroup of any point is always compact, being a subgroup of the unitary group of the metric at that point.) It is evident that

$$
\left.\bigcap_{\rho \geq \rho_{0}} \operatorname{Isom}\left(D_{\rho}, \beta\right)\right|_{K}=\left.\operatorname{Isom}(D, \beta)\right|_{K}=\left.\operatorname{Aut}_{0}(D)\right|_{K} \text {. }
$$


Since a descending family of compact Lie groups is eventually constant, this implies that $\left.\operatorname{Isom}\left(D_{\rho}, \beta\right)\right|_{K}=\left.\operatorname{Aut}_{0}(D)\right|_{K}$ for some $\rho$. Finally, since an isometry is completely determined by its behavior on a nonempty open set and $D_{\rho}$ is connected for $\rho>$ diameter $_{\beta}(K)$, we conclude that

$$
\operatorname{Aut}_{0}(D)=\operatorname{Isom}(\overline{\mathbf{D}}, \mu(\rho))=\operatorname{Isom}\left(D_{\rho}, \beta\right)
$$

for some $\rho$. We set $\mu=\mu(\rho)$. (Keep in mind that $\mu$ agrees with the product measure $\alpha^{\prime \prime}$ near $b D$.) This completes the second step.

The third step is to construct a compact, connected, smooth manifold $M$ and a metric $\gamma_{0}$ on $M$ such that $\operatorname{Aut}_{0}(D) \subset \operatorname{Isom}\left(M, \gamma_{0}\right)$. For $M$ we take the double of $\overline{\mathbf{D}}$; i.e., $M$ is the union of two copies of $\overline{\mathbf{D}}$-call them $\overline{\mathbf{D}}^{+}$and $\overline{\mathbf{D}}^{-}$, say-identified along $b D=b \overline{\mathbf{D}}^{+}=b \overline{\mathbf{D}}^{-}$. For $\gamma_{0}$ we take the obvious metric which is equal to $\mu$ on each copy of $\overline{\mathbf{D}}$ in $M$; since $\mu$ is a product metric near $b D, \gamma_{0}$ is indeed a smooth metric on $M$.

It is clear that every isometry $f \in \operatorname{Isom}(\overline{\mathbf{D}}, \mu)=\operatorname{Aut}_{0}(D)$ has a natural extension $\tilde{\mathbf{f}}$ to $M$ and that $M$ has a natural involution $\sigma$ which interchanges $\overline{\mathbf{D}}^{+}$and $\overline{\mathbf{D}}^{-}$and commutes with every $\tilde{\mathbf{f}}$. In fact, these isometries generate the entire isometry group $\operatorname{Isom}\left(M, \gamma_{0}\right)$. To see this, let $h \in \operatorname{Isom}\left(M, \gamma_{0}\right)$ and consider the image $h\left(K^{+}\right)$ of $K^{+}$under $h$. The same curvature, volume and diameter considerations as in step 2 imply that either $h\left(K^{+}\right)=K^{+}$or $h\left(K^{+}\right)=K^{-}$. If $h\left(K^{+}\right)=K^{+}$, then distance considerations, together with the fact that $\mu$ agrees with $\beta$ on $D_{\rho}$ imply that $h\left(D_{\rho}^{+}\right)=D_{\rho}^{+}$. Since $\operatorname{Isom}\left(D_{\rho}, \beta\right)=\operatorname{Aut}_{0}(D)$, this means that the restriction of $h$ to $D_{\rho}^{+}$agrees with the restriction of $\tilde{\mathbf{f}}$ to $D_{\rho}^{+}$for some $f \in \operatorname{Aut}_{0}(D)$; since an isometry is determined by its behavior on any nonempty open set, this means that $h=\tilde{\mathbf{f}}$. On the other hand, if $h\left(K^{+}\right)=K^{-}$, the same argument applied to $\sigma \circ h$ yields that $\sigma \circ h=\tilde{\mathbf{f}}$, or equivalently, that $h=\sigma \circ \tilde{\mathbf{f}}$, for some $f \in \operatorname{Aut}_{0}(D)$. This completes the third step.

The final step is to construct a small perturbation $\gamma$ of $\gamma_{0} \operatorname{such}$ that $\operatorname{Isom}(M, \gamma)=$ $G$. Recall that $\gamma_{0}$ is the double of a metric $\mu$ on $\overline{\mathbf{D}}$ which, near $b D$, is the product of an as yet unspecified $\operatorname{Aut}_{0}(D)$-invariant metric $\alpha$ on $b D$ with the Euclidean metric on $[0, \lambda)$. We first construct a specific metric $\alpha$ which will facilitate the perturbation of $\gamma_{0}$.

Recall from $\S 4$ that $\operatorname{Isom}(D, \beta)=\operatorname{Aut}_{0}(D)=\operatorname{Aut}(D) \cup \operatorname{Aut}^{*}(D)$ and that every element $f$ of $\operatorname{Aut}_{0}(D)$ extends smoothly to $\overline{\mathbf{D}}$. We will continue to denote this extension by $f$. Since $G=\operatorname{Aut}(D)$, it is clear that $\operatorname{Aut}(D)$ leaves $G$-orbits in $b D$ invariant. We claim that there is a point $x_{0} \in b D$ and a $G$-invariant neighborhood $W_{0}$ of $G \cdot x_{0}$ in $b D$ such that $f\left(W_{0}\right) \cap W_{0}=\varnothing$ for all $f \in \operatorname{Aut}^{*}(D)$. Of course, if $\operatorname{Aut}^{*}(D)$ is empty this is vacuously true. Otherwise, fix $f_{0} \in \operatorname{Aut}^{*}(D)$; we will show that for some $x_{0} \in b D$, the orbit $G \cdot x_{0}$ is not invariant under $f_{0}$, and construct $W_{0}$ from this fact.

Suppose that $f_{0}\left(G \cdot x_{0}\right) \subset G \cdot x_{0}$ for every $x_{0}=\left(z_{0}, w_{0}\right)$ in $b D$. Write $f_{0}=\left(f_{1}, f_{2}\right)$, so that $f_{1}: D \rightarrow \mathrm{GL}(n)$ and $f_{2}: D \rightarrow \mathbf{C}^{m}$ are antiholomorphic maps, and have smooth extensions to $\overline{\mathbf{D}}$. For each $x_{0}=\left(z_{0}, w_{0}\right)$ in $b D$ write

$$
\mathbf{V}_{x_{0}}=\left\{\left(Z, W_{0}\right) \in \mathrm{GL}(n) \times \mathbf{C}^{m}: Z \in \mathrm{GL}(n)\right\} \text {. }
$$

Since $G$ acts by multiplication in the first factor, $\mathbf{V}_{x_{0}} \cap b D$ is $G$-invariant, and hence invariant under $f_{0}$ (since we have supposed that $f_{0}$ leaves all $G$-orbits in $b D$ 
invariant). In particular, $f_{2}\left(z, w_{0}\right)=w_{0}$ for each $\left(z, w_{0}\right) \in \mathbf{V}_{x_{0}} \cap b D$; the identity principle then implies that $f_{2}\left(z, w_{0}\right)=w_{0}$ for each $\left(z, w_{0}\right) \in \mathbf{V}_{x_{0}} \cap D$. Thus $f_{2}(z, w)=w$ for each $(z, w) \in D$, which is absurd since this is not an antiholomorphic mapping. We conclude that there is indeed a point $x_{0} \in b D$ such that $f_{0}\left(G \cdot x_{0}\right) \not \subset G \cdot x_{0}$.

We now fix an element $g_{0} \in G$ such that $f_{0}\left(g_{0} \cdot x_{0}\right) \notin G \cdot x_{0}$. As we noted in $\S 4$, the group $\operatorname{Aut}(D)$ of holomorphic automorphisms of $D$ is normal in the group $\operatorname{Aut}_{0}(D)$ of holomorphic and antiholomorphic automorphisms; hence every left coset of $\operatorname{Aut}(D)$ is also a right coset. In particular, for each $g_{1} \in G$ we can find a $g_{2} \in G$ such that $f_{0}\left(g_{1} \cdot g_{0} \cdot x_{0}\right)=g_{2} \cdot f_{0}\left(g_{0} \cdot x_{0}\right)$. Since $f_{0}\left(g_{0} \cdot x_{0}\right) \notin G \cdot x_{0}$, we conclude that $f_{0}\left(g_{1} \cdot g_{0} \cdot x_{0}\right) \notin G \cdot x_{0}$; since $g_{1}$ is arbitrary, it follows that $f_{0}\left(G \cdot x_{0}\right) \cap G \cdot x_{0}=\varnothing$.

Finally, the continuity of $f_{0}$ on $D$ and the fact that $G \cdot x_{0}$ is $G$-invariant allow us to choose a $G$-invariant neighborhood $W_{0}$ of $G \cdot x_{0}$ in $b D$ such that $f_{0}\left(W_{0}\right) \cap W_{0}=\varnothing$. To see that $f\left(W_{0}\right) \cap W_{0}=\varnothing$ for each $f$ in $\operatorname{Aut}^{*}(D)$, note-as in $\S 4$ - that every $f \in \operatorname{Aut}^{*}(D)$ is of the form $f_{0} \circ h$ for some $h \in \operatorname{Aut}(D)$; i.e., $f(x)=f_{0}(g \cdot x)$ for some $g \in G$ (and all $x \in D$ ). But then $f\left(W_{0}\right)=f_{0}\left(g \circ W_{0}\right)=f_{0}\left(W_{0}\right)$, since $W_{0}$ is $G$-invariant, so that $f\left(W_{0}\right) \cap W_{0}=\varnothing$ for each $f \in \operatorname{Aut}^{*}(D)$. By strinking $W_{0}$ if necessary, we can assume that $W_{0}$ is a $G$-tubular neighborhood of $G \cdot x_{0}$ in $b D$; i.e., there is a diffeomorphism of $W_{0}$ with $G \times(-\varepsilon, \varepsilon)^{2 L-1-\operatorname{dim}_{\mathbf{R}}(G)}$, where $L=n^{2}+m=\operatorname{dim}_{\mathbf{C}}\left(\operatorname{GL}(n) \times \mathbf{C}^{m}\right)$, and this diffeomorphism respects the action of $G$. Let $W_{1}=\left\{f\left(W_{0}\right): f \in \operatorname{Aut}^{*}(D)\right\}$; as noted before, either $W_{1}=$ $\varnothing$ or $W_{1}=f_{0}\left(W_{0}\right)$, for $f_{0}$ a fixed element of $\operatorname{Aut}^{*}(D)$. Then $W_{2}=W_{1} \cup W_{0}$ is an $\operatorname{Aut}_{0}(D)$-tubular neighborhood of $\operatorname{Aut}_{0}(D) \cdot x_{0}$; i.e., $W_{2}$ is diffeomorphic to $\operatorname{Aut}_{0}(D) \times(-\varepsilon, \varepsilon)^{2 L-1-\operatorname{dim}_{\mathbf{R}}(G)}$ by a diffeomorphism preserving the action of $\operatorname{Aut}_{0}(D)$.

We now construct the metric $\alpha$. Let $\alpha_{1}$ be an arbitrary $\operatorname{Aut}_{0}(D)$-invariant, smooth Riemannian metric on $\operatorname{Aut}_{0}(D)$, let $\alpha_{2}$ be the Euclidean (flat) metric on $(-\varepsilon, \varepsilon)^{2 L-1-\operatorname{dim}_{\mathbf{R}}(G)}$, and let $\alpha=\alpha_{1} \times \alpha_{2}$ be the product metric on $W_{2}$. Clearly $\alpha$ is $\operatorname{Aut}_{0}(D)$-invariant on $W_{2}$. We extend $\alpha$ to an $\operatorname{Aut}_{0}(D)$-invariant metric (denoted again by $\alpha$ ) on the whole boundary $b D$.

The constructions of $\alpha, \alpha^{\prime \prime}, \mu=\mu(\rho)$, and $\gamma_{0}$ guarantee that we can find a neighborhood $Q$ of $G \cdot x_{0}$ in $M$ with all of the following properties:

(1) $Q$ is $G$-tubular; i.e., $Q=G \times(-\delta, \delta)^{N}$ and $G$ acts in the first factor (where $\left.N=2\left(n^{2}+m\right)-\operatorname{dim}_{\mathbf{R}}(G)=\operatorname{dim}_{\mathbf{R}}(M)-\operatorname{dim}_{\mathbf{R}}(G)\right)$.

(2) $\tilde{\mathbf{f}}(Q) \cap Q=\varnothing$ for all $f \in \operatorname{Aut}^{*}(D)$.

(3) $Q$ is invariant for the involution $\sigma$ and $\sigma$ acts by multiplication by -1 in the last $(-\delta, \delta)$-factor; i.e., $\sigma\left(g ; \delta_{1}, \ldots, \delta_{N-1}, \delta_{N}\right)=\left(g ; \delta_{1}, \ldots, \delta_{N-1},-\delta_{N}\right)$. In particular,

$$
\begin{aligned}
& Q \cap D^{+}=G \times(-\delta, \delta)^{N-1} \times(0, \delta), \\
& Q \cap D^{-}=G \times(-\delta, \delta)^{N-1} \times(-\delta, 0), \\
& Q \cap b D=G \times(-\delta, \delta)^{N-1} \times\{0\} .
\end{aligned}
$$

(4) On $Q$, the metric $\gamma_{0}$ is the product of the metric $\alpha_{1}$ on $\operatorname{Aut}_{0}(D)$, restricted to $G$, with the (flat) Euclidean metric on $(-\delta, \delta)^{N}$.

We now choose any point $y$ in $Q \backslash b D$; for convenience, assume

$$
y=\left(1_{G} ; 0, \ldots, 0, \delta_{N}\right)
$$


in $Q \cap D^{+}$with $0<\delta_{N}<\delta$. Let $\nu$ be any metric on $(-\delta, \delta)^{N-1} \times(0, \delta)$ which is close to the Euclidean metric but whose curvature is nonconstant on every open set and let $\eta: M \rightarrow[0,1]$ be a smooth, $G$-invariant function which is identically 1 near $G \cdot y$ and identically 0 off $Q \cap D^{+}=G \times(-\delta, \delta)^{N-1} \times(0, \delta)$. Set $\gamma=\eta\left(\alpha_{1} \times \nu\right)+(1-\eta) \gamma_{0}$. It is evident that $\gamma$ is $G$-invariant, so that $G \subset \operatorname{Isom}(M, \gamma)$; we show that the reverse inequality holds if $\nu$ is sufficiently close to the Euclidean metric (in the sense of $C^{\infty}$-closeness of metrics).

If $\nu$ is very close to the Euclidean metric then $\gamma$ is very close to $\gamma_{0}$, so Ebin's theorem implies that there is a diffeomorphism $F$ of $M$ which is close to the identity and has the property that $F^{-1} \circ h \circ F \in \operatorname{Isom}\left(M, \gamma_{0}\right)$ for every $h \in \operatorname{Isom}(M, g)$. Equivalently, the only isometries of $\gamma$ are of the form $F \circ h^{\prime} \circ F^{-1}$ with $h^{\prime} \in$ $\operatorname{Isom}\left(M, \gamma_{0}\right)$. Since $G \subset \operatorname{Isom}(M, \gamma)$, we want to show that $F \circ h^{\prime} \circ F^{-1} \notin \operatorname{Isom}(M, \gamma)$ if $h^{\prime} \in \operatorname{Isom}\left(M, \gamma_{0}\right)$ and $h^{\prime} \notin G$.

To accomplish this, it is convenient to distinguish two cases. In the first case, we suppose that $\operatorname{Aut}^{*}(D) \neq \varnothing$, and fix an element $f_{0} \in \operatorname{Aut}^{*}(D)$. Then $\tilde{\mathbf{f}}_{0}(Q) \cap Q=\varnothing$; we want to make sure that $F$ is so close to the identity that

(1) $F^{-1}(y) \in \operatorname{support}(\eta)$,

(2) $F \circ \tilde{\mathbf{f}}_{0}(\operatorname{support}(\eta)) \subset \tilde{\mathbf{f}}_{0}(Q)$,

(3) $F \circ \sigma(\operatorname{support}(\eta)) \subset Q \cap D^{-}$,

(4) $F \circ \sigma \circ \tilde{\mathbf{f}}_{0}(\operatorname{support}(\eta)) \subset \tilde{\mathbf{f}}_{0}(Q)$.

We can arrange for $F$ to have all these properties by choosing $\nu$ sufficiently close to the Euclidean metric, so that $\gamma$ is sufficiently close to $\gamma_{0}$. To see that these properties of $F$ are adequate, suppose first that $h^{\prime} \in \operatorname{Isom}\left(M, \gamma_{0}\right)$ is of the form $h^{\prime}=\tilde{\mathbf{f}}$ with $f \in \operatorname{Aut}^{*}(D)$. Just as before, there is an element $g \in G$ such that $\tilde{\mathbf{f}}(x)=\tilde{\mathbf{f}}_{0}(g \cdot x)$ for each $x \in M$. Since $h$ is a $G$-invariant function, its support is a $G$-invariant set so $\tilde{\mathbf{f}}(\operatorname{support}(\eta))=\tilde{\mathbf{f}}_{0}(\operatorname{support}(\eta))$. Since $F^{-1}(y) \in \operatorname{support}(\eta)$ and $F \circ \tilde{\mathbf{f}}_{0}(\operatorname{support}(\eta)) \subset \tilde{\mathbf{f}}_{0}(Q)$, we conclude that $F \circ \tilde{\mathbf{f}} \circ F^{-1}(y) \in \tilde{\mathbf{f}}_{0}(Q)$. Now, since $\tilde{\mathbf{f}}_{0}(Q) \cap Q=\varnothing$, the metrics $\gamma$ and $\gamma_{0}$ agree on $\tilde{\mathbf{f}}_{0}(Q)$. Because $\tilde{\mathbf{f}}_{0}$ is an isometry of $\gamma_{0}$ and the restriction of $\gamma_{0}$ to $Q$ is the product of $\alpha_{1}$ on $G$ with the flat Euclidean metric on $(-\delta, \delta)^{L}$, we conclude that at each point of $\tilde{\mathbf{f}}_{0}(Q)$ the metric $\gamma$ is of the same form. Our construction, however, guarantees that at $y$ the metric $\gamma$ is the product of $\alpha_{1}$ with a metric $\nu$ whose curvature is nonconstant on every open set. Since $F \circ \tilde{\mathbf{f}} \circ F^{-1}(y) \in \tilde{\mathbf{f}}_{0}(Q)$, this means that $F \circ \tilde{\mathbf{f}} \circ F^{-1}$ cannot be an isometry of $\gamma$ when $f \in \operatorname{Aut}^{*}(D)$, which is what we want. It remains to see what happens if $h^{\prime} \in \operatorname{Isom}\left(M, \gamma_{0}\right), h^{\prime} \notin G$, and $h^{\prime}$ is not of the form $h^{\prime}=\tilde{\mathbf{f}}$ with $f \in \operatorname{Aut}^{*}(D)$. Since $\operatorname{Isom}\left(M, \gamma_{0}\right)$ is generated by the involution $\sigma$ and by $\operatorname{Aut}_{0}(D)$, this means that $h^{\prime}=\sigma \circ \tilde{\mathbf{f}}$ for some $f \in \operatorname{Aut}_{0}(D)$. Just as above, we then conclude that $F \circ(\sigma \circ \tilde{\mathbf{f}}) \circ F^{-1}(y) \in D^{-}$; again, this means that, near $F \circ(\sigma \circ \tilde{\mathbf{f}}) \circ F^{-1}, \gamma$ is the product of $\alpha_{1}$ with a flat Euclidean metric while, near $y$, it is the product of $\alpha_{1}$ with a metric $\nu$ whose curvature is nonconstant on every open set. So $F \circ(\sigma \circ \tilde{\mathbf{f}}) \circ F^{-1}$ is not an isometry of $\gamma$. This completes the case that $\operatorname{Aut}^{*}(D) \neq \varnothing$.

In the case $\operatorname{Aut}^{*}(D)=\varnothing$, the argument is even simpler since then we need only be sure that $F^{-1}(y) \in \operatorname{support}(\eta)$ and that $F \circ \sigma(\operatorname{support}(\eta))$ is contained in $Q \cap D^{-}$; we omit the details.

In either case, we conclude that $\operatorname{Isom}(M, \gamma)=G$. This completes the proof of Theorem 1. 
It is worth noting that we could arrange for $M$ to be a real-analytic manifold, for the action of $G$ to be real-analytic, and for the metric $\gamma$ to be real-analytic. To achieve this we note, as at the end of $\S 3$, that the domain $D$ could be chosen to have real-analytic boundary; then the manifold $M$ and the action of $G$ will be real-analytic. The metric $\gamma$ constructed above need not be real-analytic, but we can replace it by a nearby $G$-invariant real-analytic metric $\tilde{\gamma}$; Ebin's theorem will guarantee that $\operatorname{Isom}(M, \tilde{\gamma})=\operatorname{Isom}(M, \gamma)=G$.

It is of interest to know the diffeomorphism type of the manifold $M$. With a little more care, we could insure that the domain $D$ is a regular neighborhood of $U(n) \times$ $\{0\}$ in $\operatorname{GL}(n) \times \mathbf{C}^{m}$, whence $M$ will be diffeomorphic to $U(n) \times S^{2 m}$. Alternatively, following the comments at the end of $\S 3$, we can arrange that the manifold $M$ we obtain will be diffeomorphic to $S^{2\left(n^{2}+m\right)}$. Note that the construction we have actually used produces an action of $G$ on $M$ which is free; if we follow the alternate procedure suggested above, we will obtain an action with a fixed point.

Finally, we should point out the crucial role played by Theorem 2 in this argument: it guarantees that all the isometries in $\operatorname{Isom}\left(M, \gamma_{0}\right)$ which do not belong to $G$ actually move some orbit of $G$, and can thus be eliminated by a small perturbation of $\gamma_{0}$.

\section{REFERENCES}

1. E. Bedford, Invariant forms on complex manifolds with application to holomorphic mappings, Math. Ann. 265 (1983), 337-397.

2. E. Bedford and J. Dadok, Bounded domains with prescribed group of automorphisms, preprint.

3. __ Bounded domains with prescribed group of automorphisms. II, preprint.

4. J.-E. Björk, Compact groups operating on Banach algebras, Math. Ann. 205 (1973), 281-297.

5. D. Burns, Jr. and S. Shnider, Real hypersurfaces in complex manifolds, Proc. Sympos. Pure Math., vol. 30, part 2, Amer. Math. Soc., Providence, R.I., 1976, pp. 141-167.

6. D. Burns, Jr., S. Shnider, and R. O. Wells, Jr., Deformations of strictly pseudoconvex domains, Invent. Math. 46 (1978), 237-253.

7. H. Cartan, Sur les fonctions de plusieurs variables complexes. L'itération des transformations intérieures d'un domaine borné, Math. Z. 35 (1932), 760-773.

8. S. S. Chern and J. K. Moser, Real hypersurfaces in complex manifolds, Acta Math. 133 (1974), 219-271.

9. __ Erratum: "Real hypersurfaces in complex manifolds" [Acta Math. 133 (1974), 219-271], Acta Math. 150 (1983), 297.

10. D. G. Ebin, The manifold of Riemannian metrics, Proc. Sympos. Pure Math., vol. 15., Amer. Math. Soc., Providence, R.I., 1970, pp. 11-40.

11. C. Fefferman, The Bergman kermel and biholomorphic mappings of pseudoconvex domains, Invent. Math. 26 (1974), 1-65.

12. M. Golubitsky and V. Guillemin, Stable mappings and their singularities, Springer-Verlag, New York, 1973.

13. R. E. Greene and S. G. Krantz, Deformations of complex structure, estimates for the $\bar{\partial}$-equation, and stability of the Bergman kernel, Adv. in Math. 43 (1982), 1-86.

14. __ The automorphism groups of strongly pseudoconvex domains, Math. Ann. 261 (1982), 425446.

15 , Normal families and the semicontinuity of isometry and automorphism groups, preprint.

16 P. F. Klembeck, Kähler metrics of negative curvature, the Bergman metric near the boundary and the Kobayashi metric on smooth bounded strictly pseudoconvex sets, Indiana Univ. Math. J. 27 (1978), 275-282.

17. S. Kobayashi, Transformation groups in differential geometry, Springer-Verlag, Berlin, 1972.

18. S. G. Krantz, Function theory of several complex variables, Wiley, New York, 1982. 
19. S. Lojasiewicz, Triangulation of semi-analytic sets, Ann. Scuola Norm. Sup. Pisa (3) 18 (1964), 449-474.

20. Q.-K. Lu, On the complete Kähler manifolds with constant unitary curvature, Acta Math. Sinica 16 (1966), 269-281; English transl., Chinese Math. 8 (1966), 283-298.

21. L. N. Mann, Gaps in the dimensions of isometry groups of Riemannian manifolds, J. Differential Geom. 11 (1976), 293-298.

22. S. B. Myers and N. E. Steenrod, The group of isometries of a Riemannian manifold, Ann. of Math. (2) 40 (1939), 400-416.

23. H. Poincaré, Les fonctions analytiques de deux variables et la représentation conforme, Rend. Circ. Mat. Palermo 23 (1907), 185-220.

24. J.-P. Rosay, Sur une caractérisation de la boule parmi les domaines de $\mathbf{C}^{n}$ par son groupe d'automorphismes, Ann. Inst. Fourier (Grenoble) 29 (4) (1979), 91-97.

25. E. M. Stein, Boundary behavior of holomorphic functions of several complex variables, Princeton Univ. Press, Princeton, N.J., 1972.

26. B. Wong, Characterization of the unit ball in $\mathbf{C}^{n}$ by it automorphism group, Invent. Math. 41 (1977), 253-257.

Department of Mathematics, Michigan State University, EAst LANSing, MICHIGAN 48824

Department of Mathematics, State University of New York at Buffalo, BUFFALO, NEW YORK 14214-3093 